\title{
Estrutura e composição da ictiofauna em um lago isolado na planície do médio rio Araguaia, Mato Grosso - Brasil
}

\author{
Elias Centofante ${ }^{1 *}$ \\ Cesar Enrique de Melo $^{2}$ \\ Universidade do Estado de Mato Grosso \\ ${ }^{1}$ Programa de Pós Graduação em Ecologia e Conservação \\ ${ }^{2}$ Laboratório de Ictiologia e Limnologia \\ Caixa Postal 08, CEP 78690-000, Nova Xavantina - MT, Brasil \\ * Autor para correspondência \\ e.centofante@bol.com.br
}

Submetido em 19/10/2011

Aceito para publicação em 28/05/2012

\section{Resumo}

Estudos sobre a ictiofauna de lagos da bacia do Araguaia, até o momento, são escassos e a maioria destes foi sobre lagos diretamente conectados ao rio e sob grande influência do pulso de inundação. Lagos isolados, com pouca influência do rio, são menos comuns na região e até agora não mereceram estudos sobre suas ictiofaunas. Este trabalho tem como objetivo discutir a estrutura da comunidade, diversidade e riqueza de espécies da ictiofauna em um lago isolado na planície do médio Rio Araguaia - MT. As amostragens foram conduzidas ao longo de dois ciclos sazonais consecutivos e resultaram em um total de 1312 peixes, distribuídos em 57 espécies, 19 famílias e seis ordens. A comunidade de peixes em estudo apresentou altos valores de diversidade. Os resultados deste trabalho sugerem que a alta transparência da água e o isolamento do lago atuam como fator primário enquanto que a estabilidade das outras variáveis abióticas atua como fator secundário.

Palavras-chave: Diversidade; Isolamento; Peixes; Transparência da água

\section{Abstract}

Fish fauna structure and composition in an isolated lake in the Middle Araguaia River floodplain, Mato Grosso - Brazil. Studies dealing with fish fauna in floodplain lakes of the Araguaia River basin are limited, and have mostly focused on lakes that are directly connected to the main river and are heavily influenced by the flood pulse. Isolated lakes are relatively uncommon in this region and have received little attention in regards to their fish fauna. This work aims to discuss the structure, diversity and richness of the fish community of an isolated lake in the floodplain of the Middle Araguaia River, MT. Samples were made through two consecutive seasonal cycles, and yielded a total of 1312 fishes, distributed in 57 species, 19 families and six orders. The studied fish community presented high values of diversity. The results of this study suggest that the high water transparency in conjunction with the isolation of the lake acts as a primary factor, while the stability of the other abiotic variables acts as a secondary factor.

Key words: Diversity; Fishes; Isolation; Water transparency 


\section{Introdução}

O sistema fluvial do rio Araguaia mantém um complexo mosaico de ambientes aquáticos lênticos e lóticos, com alta riqueza e diversidade de espécies de peixes (MÉRONA, 1986; TEJERINA-GARRO et al., 1998; LOWE-MCCONNELL, 1999).

Uma das mais importantes características dessa bacia hidrográfica é a grande quantidade de lagos, principalmente lagos de meandros, que tornam a região do médio e baixo Araguaia, uma área de grande biodiversidade de animais aquáticos, principalmente de peixes. Estudos realizados nesses lagos demonstraram que a ictiofauna é tão diversa como aquela que ocorre em ambientes similares na Amazônia Central (MELO; LIMA, 2007; SILVA et al., 2007; MELO et al., 2009). Essa diversidade é decorrente da morfometria, circulação da água e grau de isolamento dos lagos em relação ao restante do sistema, o que leva a diferenças marcantes na estrutura das assembleias de peixes. Embora, de forma geral, esses lagos da Bacia do rio Araguaia sejam predominantemente lagos de meandros, em alguns pontos da bacia ocorrem lagos isolados que podem situar-se a dezenas de quilômetros do curso principal do rio. Esses lagos, ao contrário da maioria dos lagos amazônicos, não tiveram sua gênese na ação erosiva dos grandes rios, mas estão localizados em depressões do terreno e geralmente atuam como nascentes de pequenos cursos d'água, (MENESES et al., 2007), pelos quais se ligam aos rios de maior porte.

A migração dos peixes entre um rio de grande porte e esses lagos isolados, muitas vezes é dificultada pela distância entre esses e o rio, pelo tipo de canal que os conecta e pelo próprio volume de água. Em lagos diretamente conectados ao rio esse problema inexiste. Essa condição especial torna os lagos isolados muito menos suscetíveis a alterações por imigração da ictiofauna (CLOSS et al., 2004), mantendo a mesma mais homogênea ao longo do tempo.

Além do mais, as inundações anuais dos grandes rios, que caracterizam o pulso de inundação, não afetam diretamente esses lagos isolados, em consequência suas ictiofaunas tendem a ser menos alteradas entre os períodos de cheia e seca do que àquelas de lagos de meandros, diretamente conectados ao rio (POUILLY; RODRIGUEZ, 2004). Inúmeros estudos em lagos tropicais, incluindo a bacia do rio Araguaia, demonstram que no período de cheia, ocorre intensa movimentação dos peixes entre o ambiente lótico do rio e o ambiente lêntico dos lagos de meandros, em busca de alimento, abrigos e condições de reprodução (MELO et al., 2003; HOEINGHAUS et al., 2003; MELO; LIMA, 2007). Essa movimentação altera severamente a composição da ictiofauna dos lagos entre os períodos de seca e cheia. No entanto, em lagos isolados, a precipitação pluvial na área da bacia do próprio lago é a principal força que regula o nível das águas e, portanto, esse tipo de corpo de água não está submetido ao mesmo conjunto de variações ambientais características dos lagos de meandros, que são diretamente influenciados pela variação no nível das águas dos grandes rios (JUNK et al., 1989; ESTEVES, 1998; POUILLY et al., 2004).

Uma dessas características é a grande quantidade de sedimentos das partes superiores da bacia que são drenadas para os lagos de meandros, pela ação da correnteza do rio, o que aumenta consideravelmente a turbidez das águas nesses lagos no período de cheia, conforme demonstrado por Esteves (1998), TejerinaGarro et al. (1998) e Pouilly et al. (2004).

Esse aumento na quantidade de sedimentos e, consequentemente, na turbidez da água dos lagos de meandros, também contribui para alterações na composição da ictiofauna desses ambientes, entre um período do ano e outro. Durante o período de águas mais claras, predominam espécies com maior capacidade de orientação visual, enquanto que no período de águas mais turvas, as espécies com dispositivos acessórios de orientação, como barbilhões, tendem a aumentar sua abundância (RODRÍGUEZ; LEWIS, 1997; LIMA, 2009; MELO et al., 2009).

Assim, o objetivo desse trabalho foi avaliar de forma comparativa a composição da ictiofauna e algumas condições ambientais na Baía da Saudade, um lago isolado da bacia do rio Araguaia, com estudos realizados em lagos de meandros da mesma bacia. Para tanto, foram testadas as seguintes hipóteses: 
1) As condições limnológicas em um lago isolado na bacia do rio Araguaia se mantêm mais estáveis ao longo dos períodos sazonais do que àquelas encontradas em lagos de meandros, da mesma bacia hidrográfica ou sistemas similares.

2) O isolamento da Baía da Saudade em relação ao pulso de inundação do rio Araguaia, determina menor variação na transparência da água, ao longo de um ciclo sazonal, quando comparado com lagos de meandros na mesma bacia.

3) A diversidade de peixes na Baía da Saudade é mais homogênea ao longo do ano, quando comparada com os lagos de meandros da bacia do rio Araguaia.

4) O padrão de dominância da ictiofauna na Baía da Saudade, ao nível de ordens, é mais homogêneo ao longo do ano, diferente do padrão encontrado em ambientes que sofrem influência direta da inundação dos grandes rios.

\section{Material e Métodos}

O médio curso do rio Araguaia, segundo Latrubesse e Stevaux (2006), estende-se desde a cidade de Araguaiana-MT até Conceição do Araguaia-PA em um percurso de $1.160 \mathrm{~km}$, com uma área de drenagem de $300.000 \mathrm{~km}^{2}$.

O lago Baía da Saudade, com uma área de aproximadamente 1800 ha, está situado em uma área de depressão de cerrado, no município de Cocalinho, região leste do estado de Mato Grosso (14³1'18'S, $51^{\circ} 07^{\prime} 05$ 'W). Faz parte da bacia de drenagem do rio Araguaia, ao qual se liga por meio de um corixo de aproximadamente $80 \mathrm{~km}$. Suas águas resultam do abastecimento fornecido por nascentes nas vertentes laterais da sua bacia de drenagem, lençol subterrâneo e precipitação pluvial (Figura 1).

A vegetação característica da região é o Cerrado, com formações sensu strictu e campos limpos, com predomínio de brejos e veredas colonizadas por buriti (Mauritia flexuosa). O clima predominante é o tropical com duas épocas bem definidas: um período seco (Abril a Setembro) e outro chuvoso (Outubro a Março) (AQUINO et al., 2008).

\section{Coleta de peixes}

Para coleta dos peixes foram realizadas oito campanhas de campo trimestrais de Dezembro/1999 até Setembro/2001, nos períodos de enchente (Dezembro/99 e Dezembro/00), cheia (Março/00 e Março/01), vazante (Junho/00 e Junho/01) e seca (Setembro/00 e Setembro/01). Os peixes foram capturados com auxílio de redes de emalhar do tipo monofilamento de $10 \mathrm{~m} \mathrm{x}$ $1,5 \mathrm{~m}$ e malhas de $3,4,5,6,7,8,10,12,14$ e $18 \mathrm{~cm}$ entre nós opostos, totalizando 10 redes, que foram instaladas de forma aleatória em diferentes pontos do lago, permanecendo armadas por 24 horas com revisões a cada 4 horas. Após a captura, os peixes foram fixados com solução de formalina a $10 \%$ e, posteriormente, conservados em etanol $70 \%$. Todos os peixes capturados foram tombados na coleção ictiológica da Universidade do Estado de Mato Grosso, Campus de Nova Xavantina.

\section{Variáveis limnológicas}

As variáveis ambientais foram medidas próximo à área de disposição das redes, nas mesmas datas de coleta da ictiofauna. A temperatura da água $\left({ }^{\circ} \mathrm{C}\right)$ e a condutividade $(\mu \mathrm{S})$ foram medidas com o uso de aparelho multiparâmetro microprocessado (Jenco 6350 KC). O $\mathrm{pH}$ foi medido com peagômetro eletrônico marcaWTW. $\mathrm{O}$ oxigênio dissolvido foi determinado por meio de titulação (Método de Winkler). Estas variáveis foram medidas a cada seis horas, $20 \mathrm{~cm}$ abaixo da superfície e $20 \mathrm{~cm}$ acima do fundo do lago. A transparência da água foi verificada por meio de disco de Secchi $(\mathrm{cm})$. Após a obtenção dos dados em campo, foram calculadas as médias para cada variável, em cada campanha de coletas.

\section{Análises dos dados}

A diversidade de espécies ( $\left.\mathrm{H}^{\prime}\right)$ para os diferentes períodos sazonais foi determinada pelo índice de Shannon-Wiener (MAGURRAN, 2004): $\mathrm{H}^{\prime}=-\Sigma\left(\mathrm{Pi}_{1} \log _{2}\right.$ pi), onde: pi = proporção de indivíduos encontrados de uma dada espécie.

A uniformidade foi calculada da seguinte forma: $\mathrm{U}$ $=\mathrm{H}^{\prime} / \mathrm{Hmax}$, onde: $\mathrm{U}=$ =́ndice de uniformidade, $\mathrm{Hmax}=$ $\log _{2}$ do número de espécies coletadas (MAGURRAN, 
FIGURA 1: Localização do lago Baía da Saudade na planície do médio rio Araguaia, estado de Mato Grosso, Brasil.

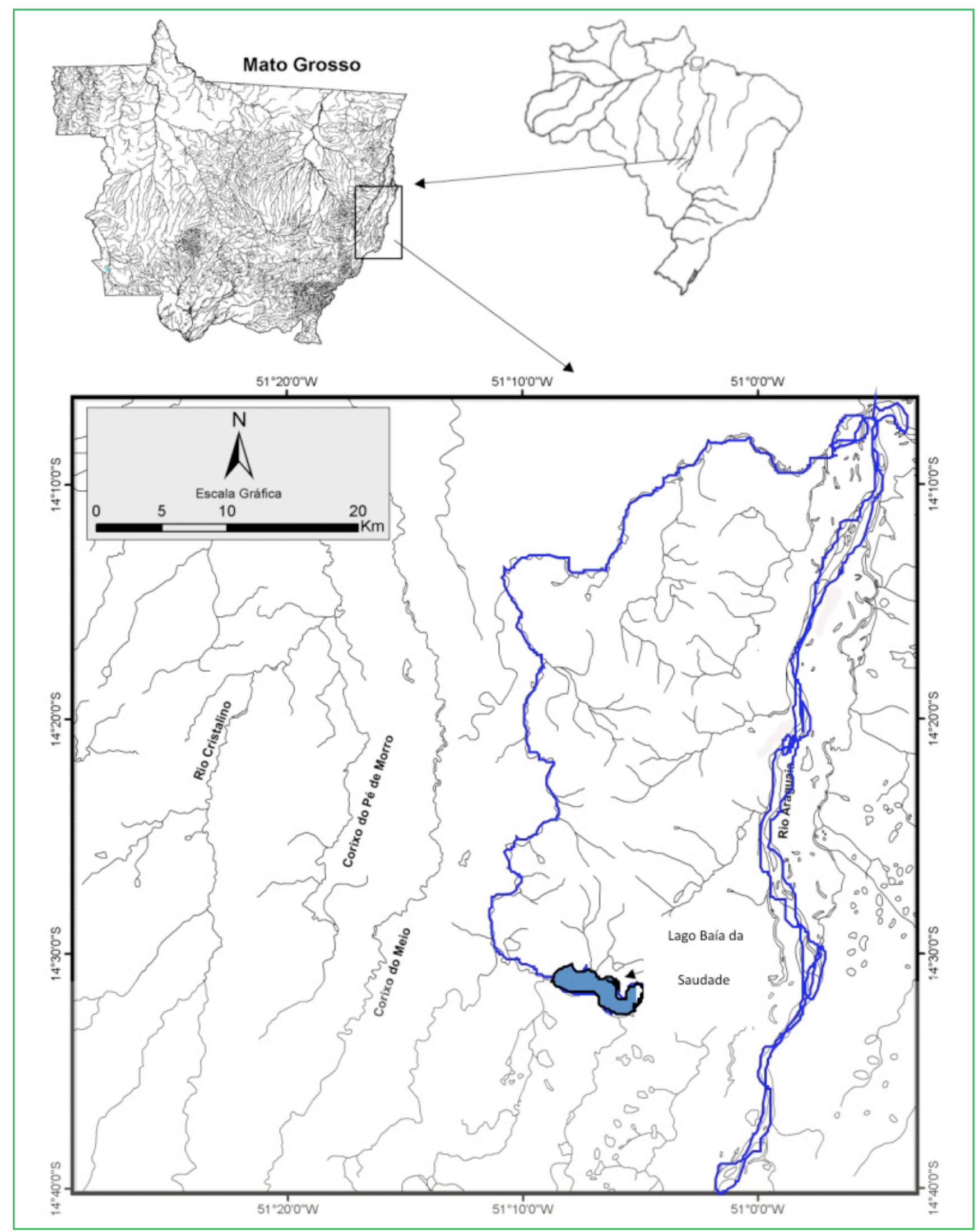


2004). Para a comparação entre os índices de diversidade de cada período, foi utilizado o teste $t$ de Student, segundo os pressupostos de Zar (1996). A dominância foi determinada como a proporção da espécie mais abundante no total coletado e a frequência de ocorrência foi calculada pela proporção do taxon no período de coletas (MAGURRAN, 2004).

A similaridade da ictiofauna entre os períodos em relação à abundância de peixes foi determinada por meio do índice de Similaridade de Morisita-Horn e a matriz de similaridade obtida foi utilizada na análise de agrupamento com uso do programa Statistica for Windows 7.0, adotando o método UPGMA (LEGENDRE; LEGENDRE, 1998).

\section{Resultados}

\section{Estrutura da ictiofauna}

Nos dois anos de coletas foram capturados um total de 1312 espécimes distribuídos em 57 espécies, 19 famílias e seis ordens. Characiformes, Perciformes e Siluriformes representaram as ordens com maior abundância e riqueza de espécies. Characiformes foi dominante em número de indivíduos em todas as coletas, com maiores abundâncias na enchente, quando representou $86,94 \%$ e na seca com $86,67 \%$ dos espécimes coletados (Figura 2).

$\mathrm{Na}$ análise geral, Characiformes também foi dominante com $80,11 \%$ do total de espécimes capturados, seguido por Perciformes, com 15,3\% e Siluriformes, com 4,04\%. As outras ordens (Gymnotiformes, Osteoglossiformes e Myliobatiformes) representam, juntas, apenas $0,55 \%$ do total coletado. Em número de espécies, os Characiformes também foram dominantes, com 35 , representando $61,4 \%$ do total coletado, seguido por Perciformes com 12 espécies e $21 \%$ das espécies coletadas.

A família Characidae foi a mais abundante, apresentando 472 indivíduos 35,98\%, seguida por Hemiodontidae com 377 indivíduos 28,73\% e Cichlidae com 201 indivíduos 15,32\% (Tabela 1). As famílias com menor abundância foram Sternopygidae, Potamotrygonidae e Doradidae com um indivíduo cada.

FIGURA 2: Abundância relativa das ordens de peixes, coletados por período sazonal no lago Baía da Saudade, planície do rio Araguaia, MT, Brasil.

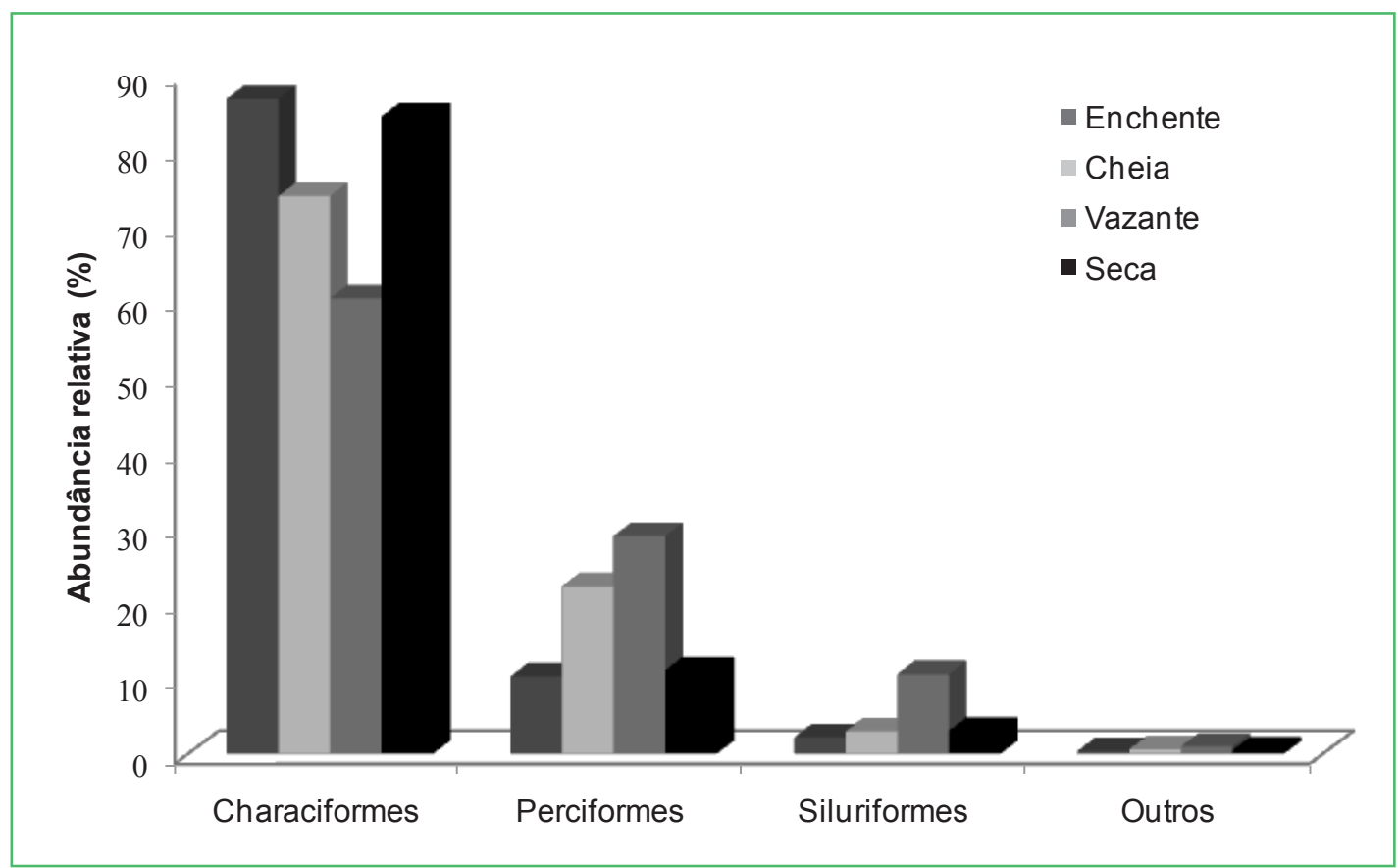


TABELA 1: Abundância das ordens, famílias e espécies, em número de indivíduos (n), e proporções relativas (\%) por período de coletas e para o total coletado no lago Baía da Saudade, planície do rio Araguaia - MT. FO = frequência de ocorrência.

\begin{tabular}{|c|c|c|c|c|c|c|c|c|c|c|c|}
\hline \multirow[t]{2}{*}{ Taxon } & \multicolumn{2}{|c|}{$\begin{array}{c}\text { Dezembro } \\
\text { Enchente }\end{array}$} & \multicolumn{2}{|c|}{$\begin{array}{l}\text { Março } \\
\text { Cheia }\end{array}$} & \multicolumn{2}{|c|}{$\begin{array}{c}\text { Junho } \\
\text { Vazante }\end{array}$} & \multicolumn{2}{|c|}{$\begin{array}{c}\text { Setembro } \\
\text { Seca }\end{array}$} & \multicolumn{2}{|c|}{ Total } & \multirow{2}{*}{$\begin{array}{c}\text { FO } \\
\%\end{array}$} \\
\hline & $n$ & $\%$ & $\mathbf{N}$ & $\%$ & $\mathbf{N}$ & $\%$ & $\mathbf{N}$ & $\%$ & $\mathbf{n}$ & $\%$ & \\
\hline Osteoglossiformes & 3 & 0,48 & & & & & 2 & 0,70 & 5 & 0,38 & \\
\hline Osteoglossidae & 3 & 0,48 & & & & & 2 & 0,70 & 5 & 0,38 & \\
\hline Osteoglossum bicirrhosum & 3 & 0,48 & & & & & 2 & 0,70 & 5 & 0,38 & 25 \\
\hline Myliobatiformes & & & 1 & 0,62 & & & & & 1 & 0,08 & \\
\hline Potamotrygonidae & & & 1 & 0,62 & & & & & 1 & 0,08 & \\
\hline Potamotrygon motoro & & & 1 & 0,62 & & & & & 1 & 0,08 & 12,5 \\
\hline Characiformes & 546 & 86,94 & 120 & 74,07 & 142 & 60,43 & 243 & 84,67 & 1051 & 80,11 & \\
\hline Curimatidae & 112 & 17,83 & 2 & 1,23 & 4 & 1,70 & & & 118 & 8,99 & \\
\hline Cyphocharax gouldingi & & & & & 1 & 0,43 & & & 1 & 0,08 & 12,5 \\
\hline Cyphocharax notatus & 2 & 0,32 & & & & & & & 2 & 0,15 & 12,5 \\
\hline Cyphocharax plumbeus & 110 & 17,52 & 2 & 1,23 & 3 & 1,28 & & & 115 & 8,77 & 50 \\
\hline Prochilodontidae & 2 & 0,32 & 1 & 0,62 & 3 & 1,28 & & & 6 & 0,46 & \\
\hline Prochilodus nigricans & 2 & 0,32 & & & & & & & 2 & 0,15 & 25 \\
\hline Semaprochilodus brama & & & 1 & 0,62 & 3 & 1,28 & & & 4 & 0,30 & 25 \\
\hline Anostomidae & 1 & 0,16 & & & 5 & 2,13 & 3 & 1,05 & 9 & 0,69 & \\
\hline Laemolyta fernandezi & & & & & 3 & 1,28 & & & 3 & 0,23 & 12,5 \\
\hline Leporinus affinis & 1 & 0,16 & & & & & & & 1 & 0,08 & 12,5 \\
\hline Leporinus friderici & & & & & 2 & 0,85 & 1 & 0,35 & 3 & 0,23 & 37,5 \\
\hline Schizodon vittatus & & & & & & & 2 & 0,70 & 2 & 0,15 & 12,5 \\
\hline Hemiodontidae & 186 & 29,62 & 63 & 38,89 & 17 & 7,23 & 111 & 38,68 & 377 & 28,73 & \\
\hline Anodus orinocensis & & & & & & & 4 & 1,39 & 4 & 0,30 & 12,5 \\
\hline Argonectes robertsi & 5 & 0,80 & 3 & 1,85 & & & & & 8 & 0,61 & 25 \\
\hline Hemiodus argenteus & 4 & 0,64 & 13 & 8,02 & 1 & 0,43 & 72 & 25,09 & 90 & 6,86 & 62,5 \\
\hline Hemiodus microlepis & 31 & 4,94 & 1 & 0,62 & & & 2 & 0,70 & 34 & 2,59 & 37,5 \\
\hline Hemiodus semitaeniatus & 2 & 0,32 & & & & & & & 2 & 0,15 & 12,5 \\
\hline Hemiodus unimaculatus & 144 & 22,93 & 46 & 28,40 & 16 & 6,81 & 33 & 11,50 & 239 & 18,22 & 100 \\
\hline Characidae & 193 & 30,73 & 30 & 18,52 & 65 & 27,66 & 65 & 22,65 & 472 & 35,98 & \\
\hline Brycon sp. & 1 & 0,16 & & & & & & & 1 & 0,08 & 12,5 \\
\hline Bryconops alburnoides & & & & & & & 1 & 0,35 & 1 & 0,08 & 12,5 \\
\hline Bryconops giacopinii & 21 & 3,34 & 8 & 4,94 & 12 & 5,11 & 3 & 1,05 & 44 & 3,35 & 75 \\
\hline Catoprion mento & 12 & 1,91 & 1 & 0,62 & 14 & 5,96 & 12 & 4,18 & 39 & 2,97 & 87,5 \\
\hline Charax leticiae & 10 & 1,59 & 1 & 0,62 & 6 & 2,55 & & & 17 & 1,30 & 62,5 \\
\hline Metynnis hypsauchen & & & & & & & 1 & 0,35 & 1 & 0,08 & 12,5 \\
\hline Moenkhausia sp. 2 & 78 & 12,42 & & & 1 & 0,43 & & & 79 & 6,02 & 25 \\
\hline Myleus sp. (juvenil) & 1 & 0,16 & 2 & 1,23 & 1 & 0,43 & 16 & 5,57 & 20 & 1,52 & 62,5 \\
\hline Poptella sp. & 38 & 6,05 & 12 & 7,41 & 5 & 2,13 & 1 & 0,35 & 56 & 4,27 & 62,5 \\
\hline Pygocentrus nattereri & 4 & 0,64 & & & 12 & 5,11 & 4 & 1,39 & 20 & 1,52 & 75 \\
\hline Serrasalmus gibbus & & & 1 & 0,62 & 4 & 1,70 & 4 & 1,39 & 9 & 0,69 & 50 \\
\hline Serrasalmus rhombeus & 28 & 4,46 & 4 & 2,47 & 10 & 4,26 & 4 & 1,39 & 46 & 3,51 & 75 \\
\hline Serrasalmus spilopleura & & & & & & & 19 & 6,62 & 19 & 1,45 & 12,5 \\
\hline Tetragonopterus chalceus & & & 1 & 0,62 & & & & & 1 & 0,08 & 12,5 \\
\hline Ctenoluciidae & 6 & 0,96 & 1 & 0,62 & 4 & 1,70 & 2 & 0,70 & 13 & 0,99 & \\
\hline Boulengerella cuvieri & & & & & & & 2 & 0,70 & 2 & 0,15 & 25 \\
\hline Boulengerella maculata & 6 & 0,96 & 1 & 0,62 & 4 & 1,70 & & & 11 & 0,84 & 37,5 \\
\hline Acestrorhynchidae & 38 & 6,05 & 13 & 8,02 & 35 & 14,89 & 52 & 18,12 & 138 & 10,52 & \\
\hline
\end{tabular}




\begin{tabular}{|c|c|c|c|c|c|c|c|c|c|c|c|}
\hline Acestrorhynchus falcirostris & 1 & 0,16 & 3 & 1,85 & 4 & 1,70 & 5 & 1,74 & 13 & 0,99 & 50 \\
\hline Acestrorhynchus microlepis & 37 & 5,89 & 10 & 6,17 & 31 & 13,19 & 47 & 16,38 & 125 & 9,53 & 100 \\
\hline Cynodontidae & 6 & 0,96 & 8 & 4,94 & & & 6 & 2,09 & 20 & 1,52 & \\
\hline Hydrolycus armatus & 6 & 0,96 & 8 & 4,94 & & & 6 & 2,09 & 20 & 1,52 & 50 \\
\hline Erythrinidae & 2 & 0,32 & 2 & 1,23 & 9 & 3,83 & 4 & 1,39 & 17 & 1,30 & \\
\hline Hoplias malabaricus & 2 & 0,32 & 2 & 1,23 & 9 & 3,82 & 4 & 1,39 & 17 & 1,30 & 75 \\
\hline Siluriformes & 14 & 2,23 & 5 & 3,09 & 25 & 10,64 & 9 & 3,14 & 53 & 4,04 & \\
\hline \multicolumn{12}{|l|}{ Loricariidae } \\
\hline Peterygoplichthys sp. & 3 & 0,48 & & & 4 & 1,70 & & & 7 & 0,53 & 37,5 \\
\hline Rineloricaria sp. & 2 & 0,32 & 1 & 0,62 & & & 1 & 0,35 & 4 & 0,30 & 50 \\
\hline Rineloricaria sp. 3 & & & & & 5 & 2,13 & & & 5 & 0,38 & 12,5 \\
\hline Pimelodidae & & & & & 3 & 1,28 & & & 3 & 0,23 & \\
\hline Hemisorubim platyrhynchos & & & & & 1 & 0,43 & & & 1 & 0,08 & 12,5 \\
\hline Pseudoplatystoma punctifer & & & & & 2 & 0,85 & & & 2 & 0,15 & 12,5 \\
\hline Doradidae & 1 & 0,16 & & & & & & & 1 & 0,08 & \\
\hline Anadoras sp. & 1 & 0,16 & & & & & & & 1 & 0,08 & 12,5 \\
\hline Auchenipteridae & 8 & 1,27 & 4 & 2,47 & 13 & 5,53 & 8 & 2,79 & 33 & 2,52 & \\
\hline Auchenipterichthys coracoideus & 8 & 1,27 & 4 & 2,47 & 13 & 5,53 & 8 & 2,79 & 33 & 2,52 & 87,5 \\
\hline Gymnotiformes & & & & & & & 1 & 0,35 & 1 & 0,08 & \\
\hline Sternopygidae & & & & & & & 1 & 0,35 & 1 & 0,08 & \\
\hline Sternopygus sp. & & & & & & & 1 & 0,35 & 1 & 0,08 & 12,5 \\
\hline Perciformes & 65 & 10,35 & 36 & 22,22 & 68 & 28,94 & 32 & 11,15 & 201 & 15,32 & \\
\hline Cichlidae & 65 & 10,35 & 36 & 22,22 & 68 & 28,94 & 32 & 11,15 & 201 & 15,32 & \\
\hline Biotodoma sp. & 12 & 1,91 & & & 1 & 0,43 & 2 & 0,70 & 15 & 1,14 & 37,5 \\
\hline Cichla monoculus & & & & & & & 3 & 1,05 & 3 & 0,23 & 12,5 \\
\hline Cichla ocellaris & 3 & 0,48 & 5 & 3,09 & 5 & 2,13 & & & 13 & 0,99 & 50 \\
\hline Cichla sp. & & & & & 1 & 0,43 & & & 1 & 0,08 & 12,5 \\
\hline Cichla temensis & 5 & 0,80 & 12 & 7,41 & 17 & 7,23 & & & 34 & 2,59 & 62,5 \\
\hline Crenicichla johanna & 1 & 0,16 & & & & & 1 & 0,35 & 2 & 0,15 & 25 \\
\hline Crenicichla lugubris & & & & & 1 & 0,43 & & & 1 & 0,08 & 12,5 \\
\hline Crenicichla sp. (juvenil) & & & & & 1 & 0,43 & & & 1 & 0,08 & 12,5 \\
\hline Crenicichla strigata & & & 1 & 0,62 & 3 & 1,28 & & & 4 & 0,30 & 25 \\
\hline Geophagus surinamensis & 20 & 3,18 & 15 & 9,26 & 31 & 13,19 & 21 & 7,32 & 87 & 6,63 & 100 \\
\hline Mesonauta acora & 2 & 0,32 & & & 1 & 0,43 & & & 3 & 0,23 & 25 \\
\hline Satanoperca pappaterra & 22 & 3,50 & 3 & 1,85 & 7 & 2,98 & 5 & 1,74 & 37 & 2,82 & 62,5 \\
\hline TOTAL & 628 & 100 & 162 & 100 & 235 & 100 & 287 & 100 & 1312 & 100 & \\
\hline
\end{tabular}

As espécies mais abundantes nas coletas foram: Hemiodus unimaculatus (Hemiodontidae) com 239 indivíduos (18,22\%), Acestrorhynchus microlepis (Acestrorhynchidae) com 125 indivíduos (9,53\%), Cyphocharax plumbeus (Curimatidae) com 115 indivíduos $(8,77 \%)$ e Hemiodus argenteus (Hemiodontidae) com 90 peixes $(6,86 \%)$.

A diversidade de espécies para o conjunto de quatro períodos de amostragem foi de $\mathrm{H}^{\prime}=4,44$. Na análise por períodos, a maior diversidade foi em Junho, período de vazante, com $\mathrm{H}^{\prime}=4,38$, período em que foram obtidos também os maiores valores de riqueza e uniformidade. A menor diversidade foi em Setembro, período de seca, com $H^{\prime}=3,76$. Apesar da variação nos valores, o teste $\boldsymbol{t}$ de student não indicou diferenças significativas entre os períodos sazonais (Tabela 2). Em relação à uniformidade, na vazante se verificou o maior valor $(U=0,848)$, já, para o período enchente obteve-se o menor valor $U=0,736$. 
TABELA 2: Variação no número de espécies (S), indivíduos $(\mathrm{N})$, diversidade (H'), uniformidade (U) da ictiofauna do lago Baía da Saudade, durante os oito períodos de coleta e no período total.

\begin{tabular}{lcccc}
\hline Período & H' & S & N & U \\
\hline Enchente & 3,77 & 35 & 628 & 0,736 \\
Cheia & 3,79 & 27 & 161 & 0,799 \\
Vazante & 4,38 & 36 & 236 & 0,848 \\
Seca & 3,76 & 31 & 287 & 0,760 \\
\hline Total & $\mathbf{4 , 4 4}$ & $\mathbf{5 7}$ & $\mathbf{1 3 1 2}$ & $\mathbf{0 , 7 6 2}$ \\
\hline
\end{tabular}

\section{Variáveis ambientais}

A temperatura da água no lago apresentou uma média de $27,1^{\circ} \mathrm{C}$, com coeficiente de variação (CV) de $8,46 \%$, sendo que o maior valor foi registrado em Dezembro/99 $\left(29,8^{\circ} \mathrm{C}\right)$ e o menor em Junho/01 $\left(23,4^{\circ} \mathrm{C}\right)$. A temperatura média da água da superfície e do fundo se manteve relativamente homogênea ao longo dos períodos de coleta. A transparência da água, com exceção de Setembro/00, foi total para todos os períodos. A condutividade apresentou baixos valores médios durante os dois anos, com mínima de $8,7 \mu \mathrm{S} . \mathrm{cm}^{-1}$ e máxima de 14,6 $\mu \mathrm{S} . \mathrm{cm}^{-1}$ e CV de 19,94\% para os períodos de estudo.

Quanto ao $\mathrm{pH}$, os valores permaneceram alcalinos durante todos os períodos com média de 7,96 e CV de $5,49 \%$. A concentração de oxigênio dissolvido apresentou variação entre 4,1 e 7,5mg.L.-1 e CV de 16,81\%, mostrando altos níveis ao longo dos períodos sazonais. A profundidade máxima registrada foi de $230 \mathrm{~cm}$, em
Março/00, e mínima, em Junho/01, com $103 \mathrm{~cm}$. A média em $177 \mathrm{~cm}$ e o CV em $24,84 \%$ (Tabela 3 ).

\section{Similaridade da ictiofauna}

A análise de agrupamento da ictiofauna entre os períodos sazonais demonstrou a ocorrência de dois grupos formados por períodos de cheia e seca, separados por período anual, o primeiro agrupou Março/00, Dezembro/00 e Setembro/00. O segundo grupo é formado por Junho/00, Março/01, Junho/01 e Setembro/01. Já Dezembro/99 se apresenta isolado de todos os outros períodos (Figura 3).

Entre todas as espécies, somente 13 foram coletadas em todos os períodos, o que representou $22,80 \%$ do total. Algumas espécies foram coletadas em um único período, e outras tiveram grandes flutuações na abundância, como Cyphocharax plumbeus que foi a segunda espécie em ordem de dominância em Dezembro/99 e esteve ausente ou com abundância muito baixa nos outros meses de coleta.

\section{Comparação entre ambientes}

Os resultdos da comparação das variáveis limnológicas e riqueza de espécies de peixes obtidos na Baía da Saudade e de alguns lagos já estudados na bacia Amazônica e do rio Araguaia, indicam características distintas entre um lago isolado e aqueles sujeitos ao pulso de inundação, em relação a vários parâmetros, entre estes, a profundidade e a transparência da água parecem

TABELA 3: Valores por períodos de coletas e média geral das principais variáveis limnológicas do lago Baía da Saudade. Temperatura (Tp), Condutividade elétrica (Cd), Oxigênio dissolvido (OD), Potencial de Hidrogênio (pH), Profundidade (Prof) e Transparência (Transp). O coeficiente de variação (CV) é dado entre parênteses.

\begin{tabular}{lcccccc}
\hline Períodos & Tp $\left({ }^{\mathbf{}} \mathbf{C}\right)$ & $\mathbf{C d}\left(\boldsymbol{\mu S . \mathbf { c m } ^ { - 1 } )}\right.$ & $\mathbf{O D}\left(\mathbf{m g ~ \mathbf { ~ } ^ { - 1 } )}\right.$ & $\mathbf{p H}$ & Prof $(\mathbf{c m})$ & Transp $(\mathbf{c m})$ \\
\hline Dezembro/99 & 29,8 & 8,7 & 4,1 & 8,4 & 181 & 181 \\
Março/00 & 29,6 & 14,4 & 6,4 & 7,6 & 230 & 230 \\
Junho/00 & 26,5 & 14,6 & 6,7 & 7,6 & 129 & 129 \\
Setembro/00 & 26,4 & 9,2 & 7,5 & 8,0 & 186 & 174 \\
Dezembro/00 & 26,1 & 11,0 & 7,1 & 7,5 & 179 & 179 \\
Março/01 & 29,6 & 10,6 & 5,7 & 7,7 & 230 & 230 \\
Junho/01 & 23,4 & 10,8 & 7,0 & 8,2 & 103 & 103 \\
Setembro/01 & 25,8 & 9,8 & 6,9 & 8,7 & 176 & 176 \\
\hline Média & $\mathbf{2 7 , 1}$ & $\mathbf{1 1 , 1}$ & $\mathbf{6 , 4}$ & $\mathbf{7 , 9 6}$ & $\mathbf{1 7 7}$ & $\mathbf{1 7 5}$ \\
(CV) & $\mathbf{( 8 , 4 6 )}$ & $\mathbf{( 1 9 , 9 4 )}$ & $\mathbf{( 1 6 , 8 1 )}$ & $\mathbf{( 5 , 4 9 1 )}$ & $\mathbf{( 2 4 , 8 4 )}$ & $\mathbf{( 2 4 , 9 6 )}$ \\
\hline
\end{tabular}


FIGURA 3: Dendograma de similaridade ictiofaunística do lago Baía da Saudade com base nos índices de Morisita-Horn (UPGMA) para os períodos de coleta em relação á abundância da ictiofauna.

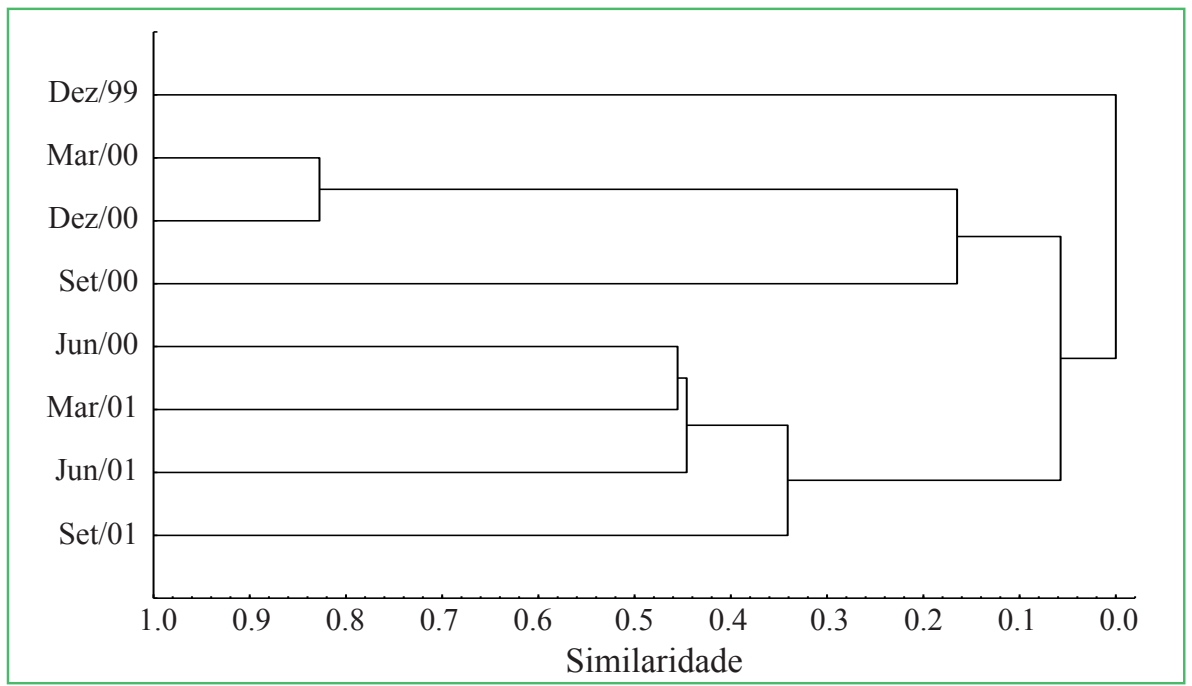

ser os mais importantes, a riqueza de espécies de peixes está dentro do esperado para ambientes lênticos dessas bacias hidrográficas (Tabela 4).

\section{Discussão}

\section{Variáveis ambientais}

Em relação aos dados ambientais as temperaturas registradas no lago Baía da Saudade foram muito próximas às encontradas em outros ambientes lênticos da Bacia Araguaia-Tocantins ou Amazônica (RODRIGUEZ; LEWIS, 1997; TEJERINA-GARRO et al., 1998; POUILLY; RODRIGUEZ, 2004; GRANADOLORENCIO et al., 2005; SILVA et al., 2007; CORREA, 2008; LIMA, 2009).

Quanto à distribuição de oxigênio dissolvido os valores permaneceram com pouca variação ao longo dos períodos com média de $6,4 \mathrm{mg} . \mathrm{L}^{-1}$ e $\mathrm{CV}$ de 16,81 , com exceção de Dezembro/99 quando ocorreu o menor valor $\left(4,10 \mathrm{mg} . \mathrm{L}^{-1}\right)$, provavelmente como consequência

TABELA 4: Amplitude de variação de variáveis limnológicas e riqueza de espécies de peixes na Baía da Saudade e outros lagos Sul americanos, com base em dados secundários. Temperatura $\left({ }^{\circ} \mathrm{C}\right)$; Condutividade elétrica $\left(\mu \mathrm{S} . \mathrm{cm}^{-1}\right)$; Oxigênio dissolvido $\left(\mathrm{mg} \cdot \mathrm{L}^{-1}\right)$; Transparência $(\mathrm{cm})$; Profundidade $(\mathrm{cm})$ e $\mathrm{pH}$.

\begin{tabular}{|c|c|c|c|c|c|c|c|c|}
\hline Variáveis & $\begin{array}{l}\text { Baia da } \\
\text { Saudade }\end{array}$ & $\begin{array}{c}\text { Lago } \\
\text { Tartaruga - } \\
\text { MT } \\
(\text { Lima, 2009) }\end{array}$ & $\begin{array}{c}\text { Lago Taraira } \\
\text { - Colômbia } \\
\text { (Correa, } \\
\text { 2008) }\end{array}$ & $\begin{array}{l}\text { Lagos no } \\
\text { Amazonas } \\
\text { (Granado- } \\
\text { Lorencio et } \\
\text { al., 2005) }\end{array}$ & $\begin{array}{c}\text { Lagos no rio } \\
\text { Araguaia } \\
\text { (Tejerina-Garro } \\
\text { et al., 1998) }\end{array}$ & $\begin{array}{c}\text { Lagos na } \\
\text { Bolívia } \\
\text { (Pouilly e } \\
\text { Rodriguez, } \\
2004 \text { ) } \\
\end{array}$ & $\begin{array}{c}\text { Lagos na } \\
\text { Venezuela } \\
\text { (Rodriguez } \\
\text { e Lewis, } \\
\text { 1997) } \\
\end{array}$ & $\begin{array}{c}\text { Enseada } \\
\text { no Rio das } \\
\text { Mortes } \\
\text { (Silva et al., } \\
2007) \\
\end{array}$ \\
\hline pH & $7,5-8,7$ & $60-63$ & $5,4-5,6$ & * & $6,6-7,3$ & $6,0-7,8$ & $6,0-6,0$ & $6,32-6,82$ \\
\hline Temperatura & $23,4-$ & $28,4-28,6$ & $27,7-28,5$ & $26,7-28,6$ & & $25,9-29,1$ & $*$ & 26,4 \\
\hline Condutividade & $8,7-14,6$ & $7,7-9,5$ & $*$ & $47,0-206,0$ & 3,6 & $16,0-228,0$ & $30,5-420,0$ & $9,4-11,4$ \\
\hline $\begin{array}{l}\text { Oxigênio } \\
\text { dissolvido }\end{array}$ & $4,1-7,5$ & $28-66$ & 85 & $1,15-4,19$ & 1,5 & $*$ & $7,0-12,3$ & $4,10-7,9$ \\
\hline Transparência & Total & $102,0-132,5$ & $70,0-124,0$ & * & 132,0 & $8,5-77,5$ & $22,5-130,0$ & $63,5-130,5$ \\
\hline Profundidade & $103-230$ & $412-671$ & $130-500$ & $*$ & $100-660$ & $60-870$ & $150-460$ & $165-500$ \\
\hline Riqueza & $27-36$ & $25-61$ & $21-44$ & $*$ & $28-50$ & * & $*$ & $22-52$ \\
\hline
\end{tabular}

* Dados não coletados ou não informados pelos autores. 
do aumento da temperatura da água que atingiu o máximo neste período, afetando a solubilidade do oxigênio na água (ESTEVES,1998). Valores diferentes foram encontrados em ambientes de lagos de meandros amazônicos que apresentaram grande variação de amplitudes entre os períodos sazonais. GranadoLorencio et al. (2007) observaram média de 1,08 mg.L $\mathrm{L}^{-1} \mathrm{e}$ CV de 52,0\% e Lima (2009) obteve média de 4,7mg.L.-1 e CV de $42,8 \%$, tendo grande aumento do oxigênio dissolvido na época seca devido a maior transparência da água, que favorece a produção dos organismos fotossintéticos e forte queda na época chuvosa, devido à turbidez e decomposição na coluna da água ocasionado pela ação do pulso de inundação dos rios (JUNK et al., 1989). Neste caso, devido ao isolamento, o lago Baía da Saudade mantém-se mais estável ao longo dos períodos sazonais, provavelmente por não ter a influência da entrada de águas do rio, que no período de cheia chegam carregados de sedimentos e, em muitos casos, com baixos níveis de oxigênio dissolvido.

Os baixos valores de condutividade encontrados no lago Baía da Saudade, estão de acordo com o esperado para lagos da bacia do Araguaia. No entanto, esses valores mais baixos, estão mais próximos daqueles observados para lagos associados ao Rio das Mortes do que ao Araguaia (TEJERINA-GARRO, 1998; LIMA, 2003; 2009). O Rio das Mortes é reconhecidamente um rio de águas mais claras que o rio Araguaia, e, portanto, os lagos associados ao Rio das Mortes, no período de cheias, recebem águas com menos nutrientes que os lagos associados ao Araguaia. No caso do Lago Baía da Saudade, é possível que o isolamento, que impede a entrada de nutrientes carreados por um grande rio mantenha o baixo nível de íons dissolvidos na água e em consequência, a baixa condutividade.

Em relação aos valores de $\mathrm{pH}$, houve uma evidente discrepância entre o Lago Baía da Saudade e os que foram comparados a este. De forma geral, os outros lagos apresentaram valores levemente ácidos ou próximos ao neutro. Valores unicamente alcalinos só foram encontrados na Baía da Saudade. Uma das causas prováveis para isso é a presença de rochas calcárias no subsolo, que são relativamente comuns na região. No entanto, não se pode descartar a possibilidade de contaminação por infiltração, de corretivos agrícolas, aplicados nas pastagens em praticamente todo o entorno do lago. Esses valores de $\mathrm{pH}$ alcalinos, também foram relativamente estáveis ao longo dos dois anos de coletas. As alterações de profundidade na Baía da Saudade, também demonstram uma maior homogeneidade do Lago quando comparados a outros que sofrem impacto do pulso de inundação. Esse parâmetro variou entre 103 e $230 \mathrm{~cm}$, enquanto que ambientes próximos aos grandes rios, com maior influência do pulso, essa variação é muito maior (CORREIA, 2008; LIMA, 2009). Situações semelhantes foram encontradas por Pouilly et al. (2004) em lagos isolados de savana da Bacia do rio Mamoré, enquanto que em lagos associados diretamente aos rios, os mesmos autores registraram variações de até $400 \mathrm{~cm}$ na profundidade de lagos, entre a seca e a cheia. Em lagos conectados ao Rio Solimões, Sousa e Freitas (2008) encontraram variações de $480 \mathrm{~cm}$. Tejerina-Garro et al. (1998) verificaram alterações entre 200 e $460 \mathrm{~cm}$ no nível da água em lagos de meandro do médio rio Araguaia. Lima (2009) em lagos da Bacia do Rio das Mortes também obteve variações entre 260 e $370 \mathrm{~cm}$.

A análise comparativa dos dados obtidos na Baía da Saudade com lagos diretamente associados a grandes rios, demonstram que a ausência de influência direta do pulso de inundação, pela falta da entrada de água de um grande rio no período de chuvas, determina uma maior estabilidade das variáveis ambientais de lagos isolados ao longo do tempo.

\section{Variação temporal da ictiofauna}

Estudos realizados por Pouilly e Rodríguez (2004) demonstram que lagos isolados de savanas sofrem menos influência do pulso de inundação do que lagos associados às planícies alagáveis de grandes rios, os quais estão sujeitos a graus distintos de conectividade hidrológica (ligado direto ou sazonalmente ao rio). Sendo amplamente regulados pela época e intensidade das cheias, criando importantes áreas para os peixes e possibilitando a migração lateral da ictiofauna (JUNK et al., 1989; TEJERINA-GARRO et al., 1998; POUILLY et al., 2004). 
Na região do médio rio Araguaia, os maiores picos da cheia geralmente acontecem entre Janeiro e Abril. Neste período, a vazão do rio aumenta de 8 a 13 vezes o volume, quando comparado ao período de seca, e esse grande volume de água é armazenado temporariamente na planície aluvial, o que é favorecido pela existência de um complexo sistema de lagos e pela saturação da planície aluvial do rio (LATRUBESSE; STEVAUX, 2006). Essa inundação sazonal provoca grandes mudanças nas características internas dos lagos de meandro, causando forte alteração na estrutura da ictiofauna ao longo dos períodos sazonais (TEJERINA-GARRO et al., 1998; POUILLY et al., 2004; POUILLY; RODRÍGUEZ, 2004; GRANADO-LORENCIO et al., 2007; LIMA, 2009).

O lago Baía da Saudade reúne condições contrárias àquelas observadas para esses tipos de lagos de meandro. Ele é afetado de forma menos intensa pelas inundações periódicas do pulso de inundação, pelo fato de estar localizado distante de um grande rio no ambiente plano da savana, pelo seu corpo hídrico ser originado numa depressão do terreno com o afloramento do lençol freático e por ter sua profundidade pouco alterada pela época da cheia.

O complexo mosaico de unidades morfosedimentares da Bacia do rio Araguaia contribui para que a planície aluvial desse sistema seja um local de grande riqueza de espécies (MÉRONA, 1986; TEJERINAGARRO et al., 1998; MELO et al., 2005). No caso do lago Baía da Saudade, embora seja um lago relativamente isolado, inserido nesse sistema, a riqueza de 57 espécies demonstra que esse ambiente com menor influência de distúrbios hidrológicos sazonais, típicos dos lagos de meandro, mantêm a tendência de um número elevado de espécies, característico dos lagos da Bacia.

Além disso, a riqueza encontrada nesse lago também é próxima àquelas encontradas em lagos associados a outros sistemas, como Rio Amazonas onde Granado-Lorencio et al. (2007) encontraram entre 54 e 79 espécies. Siqueira-Souza e Freitas (2004) descrevem riquezas entre 41 e 50 espécies em lagos da Bacia do Rio Solimões. Em um lago de meandros da planície de inundação do Rio das Mortes, Lima (2003) coletou 79 espécies e Correa (2008) descreve 53 espécies para um lago da Amazônia Colombiana.
O número de espécies também apresentou pouca variação sazonal, sendo 27 na cheia (março) e 31 na seca (setembro), e isso pode estar associado à baixa influência do pulso de inundação (JUNK et al., 1989).

O valor do índice de diversidade no lago Baía da Saudade foi de 4,44, quando considerado o total amostrado. A diversidade de espécies está dentro dos limites de diversidades encontradas em ambientes aquáticos amazônicos, que de forma geral, apresentam valores entre $\mathrm{H}^{\prime}=0,82 \mathrm{e} \mathrm{H}=4,44$ (SANTOS; FERREIRA, 1999; SILVANO et al., 2000; SIQUEIRASOUZA; FREITAS, 2004; LIN; CARAMASCHI, 2005). Esse valor também se aproxima aos valores observados em outros lagos da Bacia Araguaia-Tocantins, entre $\mathrm{H}=3,23$ e $\mathrm{H}=5,10$ (MÉRONA, 1986; LIMA, 2003; MELO; LIMA 2007; SILVA et al., 2007; MELO et al., 2007).

De modo geral, a composição da ictiofauna ao longo do ciclo sazonal se manteve relativamente homogênea. Na análise sazonal, a diversidade de espécies entre os períodos apresentou valores próximos com $H^{\prime}=3,76$ na seca (setembro) e $H^{\prime}=3,79$ na cheia (março) e com valor de uniformidade médio elevado $(0,762)$ mostrando uma distribuição homogênea no número de indivíduos entre as espécies. Resultados diferentes foram encontrados em um lago de meandro da Bacia do Rio das Mortes por Lima (2003) que obteve acentuado aumento na riqueza e diversidade de espécies na época da cheia. Neste período, a autora explica que a conexão mais ampla com o rio facilita os movimentos migratórios dos peixes que buscam recursos nas áreas laterais deste. Tejerina-Garro et al. (1998) também observaram acentuada variação na estrutura da ictiofauna em lagos diretamente associados ao médio rio Araguaia; no entanto, relacionada com a variação da transparência da água, sendo que na cheia, com águas mais turvas, ocorreu uma retração na abundância dos Characiformes e um aumento de Siluriformes.

A grande abundância de peixes das famílias Characidae e Hemiodontidae, no lago Baía da Saudade, também pode ser explicada pelos seus atributos ecológicos, como orientação primariamente visual, que são favorecidos em ambientes lênticos de elevadas transparências (LIN; CARAMASCHI, 2005; MELO 
et al., 2005), tendo à espécie Hemiodus unimaculatus dominado as capturas no lago. A ocorrência da ordem Perciformes como a segunda ordem mais abundante, diverge da maioria dos estudos em ambientes lênticos amazônicos (TEJERINA-GARRO et al., 1998; SIQUEIRA-SOUZA; FREITAS 2004; POUILLY et al., 2004; CORREA et al., 2008), o que pode ser explicado pela menor influência do pulso de inundação sobre esse ambiente, que mantém alta transparência da água, por falta da entrada de materiais particulados e dissolvidos provenientes das águas do rio Araguaia, o que beneficia também esse grupo de peixes (TEJERINA-GARRO et al., 1998; LIMA, 2009; MELO et al., 2009). Isso justifica a maior abundância de Hemiodus unimaculatus na Baía da Saudade, uma espécie predominante em ambientes de águas com elevada transparência.

Pouilly e Rodríguez (2004) obtiveram resultados semelhantes em dois lagos isolados de savana na Bacia do Rio Mamoré, na Bolívia onde obtiveram $7,26 \%$ de Siluriformes, próximos portanto, aos valores encontrados na Baía da Saudade e $88,6 \%$ de Characiformes. Os autores explicam que o grau de conexão e a alta transparência da água, associada com sua maior estabilidade nas condições internas, favorecem os Characiformes que são mais bem adaptados a essas condições. Fato corroborado por Melo et al. (2009), que demonstraram forte influência da transparência da água sobre Cynodontidae.

Hemiodus unimaculatus foi a espécie mais abundante na Baía da Saudade, com abundância relativa de $18,22 \%$. Este resultado está dentro dos valores encontrados na Bacia Amazônica por Santos e Ferreira (1999) para algumas espécies dominantes, as quais apresentam abundância relativa oscilando entre 15 a $30 \%$ e próximo aos valores observados na Bacia do Araguaia, entre 16 a 29\% (TEJERINA-GARRO et al., 1998; LIMA, 2003; MELO et al., 2007).

Com a baixa dominância, os valores de uniformidade encontrados na Baía da Saudade podem ser considerados elevados, variando entre 0,736 e 0,848 , já que a média da uniformidade em situações de alta diversidade está próximo de 80\% (ODUM, 1988). Estes valores estão de acordo com outros trabalhos da Bacia do Araguaia onde Lima (2003) obteve $72 \%$ e Silva et al. (2007) encontraram $81 \%$. Este padrão de poucas espécies dominantes, com muitos indivíduos distribuídos entre as espécies raras ou pouco abundantes, é uma característica das ictiofaunas tropicais. Essa também é uma característica das comunidades de peixes da Bacia Amazônica, onde somente em ambientes alterados ou em momentos de migração são observadas espécies com dominância maior que 30\% (SANTOS; FERREIRA, 1999).

A elevada transparência da coluna d'água aparenta ser o principal atributo ecológico selecionador da ictiofauna do lago, visto que, as duas principais ordens Characiformes e Perciformes, grupos compostos em sua maioria por peixes com orientação primariamente visual, compõem $95,43 \%$ da abundância total de peixes. Esse padrão de constituição da ictiofauna é mantido ao longo dos períodos, ao contrário dos lagos de meandros em que a inundação sazonal altera a estrutura da ictiofauna com forte aumento de Siluriformes, onde predominam espécies com sistemas de orientação acessórios, como barbilhões, na época da cheia, com águas mais turvas, como demonstrado na Bacia Amazônica por GranadoLorencio et al. (2005), no Pantanal por Súarez et al. (2004) e na Bacia do Araguaia (TEJERINA-GARRO et al., 1998; MELO et al., 2007; LIMA, 2009).

\section{Similaridade e sazonalidade}

Em lagos associados à planície de grandes rios que sofrem a influência do pulso de inundação, é característico que ocorra acentuada variação sazonal na composição e abundância da ictiofauna (JUNK et al., 1989). Comparações de similaridade e estrutura da ictiofauna entre ambientes lênticos associados à planície de inundações têm sido realizadas em lagos sul americanos (PETRY et al., 2003; LIMA, 2003; POUILLY; RODRIGUES, 2004; SÚAREZ et al., 2004; POUILLY et al., 2004) e em lagos de zonas temperadas na América do Norte (TONN; MAGNUSON, 1982; WINEMILLER et al., 2000). Esses lagos mostraram um padrão de distribuição em relação à composição e estrutura da ictiofauna, de acordo com as variações sazonais típicas desses ambientes que sofrem a ação do pulso de inundação (JUNK et al., 1989). 
No entanto, a ictiofauna do Lago Baía da Saudade se comporta de forma diferente da observada em lagos diretamente influenciados por grandes rios de planícies inundáveis e, não demonstra a ocorrência de substituição da ictiofauna entre os períodos de cheia e seca, como relatado para esses ambientes.

Este estudo demonstra que os altos índices de diversidade e riqueza do lago Baía da Saudade, apesar deste ser isolado, estão dentro do esperado para lagos conectados ou isolados da bacia amazônica ou do Araguaia. No entanto, algumas evidências neste estudo sugerem que a elevada predominância de Characiformes e Perciformes, associada a baixa representatividade de Siluriformes, parece ser efeito da elevada transparência da água, que se mantém ao longo de todo o ano.

Esses lagos, pouco conhecidos, embora comuns no bioma Cerrado, devem merecer mais atenção de políticas ambientais, já que na maioria dos casos, estão em áreas de intensa atividade agropecuária e, portanto, sob forte pressão de degradação do bioma. A conservação desses ambientes significa a manutenção de uma ictiofauna com estrutura singular e de grande importância para a diversidade regional.

\section{Agradecimentos}

À equipe do laboratório de Ictiologia e Limnologia da Universidade do Estado de Mato Grosso-UNEMAT, Campus de Nova Xavantina, pelo auxílio na realização deste trabalho, e à FAPEMAT e PROCAD/CAPES pelo apoio financeiro.

\section{Referências}

AQUINO, S.; LATRUBESSE, E. M.; SOUZA FILHO, E. E. Relações entre o regime hidrológico e os ecossistemas aquáticos da planície aluvial do Rio Araguaia. Acta Scientiarum. Biological Sciences, Maringá, v. 30, n.4, p. 361-369, 2008.

CLOSS, G.; DOWNES, B.; BOULTON, A. Freshwater ecology: a scientific introduction. Oxford: Blackwell, 2004. 225 p.

CORREA, S. B. Fish assemblage structure is consistent through an annual hydrological cycle in habitats of a floodplain-lake in the Colombian Amazon. Neotropical Ichthyology, Porto Alegre, v. 6 n. 2, p. 257-266, 2008.

CORREA, S. B.; CRAMPTON, W. G. R.; CHAPMAN, L. J.; ALBERT, J. S. A comparison of flooded forest and floating meadow fish assemblages in an upper Amazon floodplain. Journal of Fish Biology, Maiden, v. 72, n. 3, p. 629-644, 2008.

ESTEVES, F. A. Fundamentos de Limnologia. Rio de Janeiro: Interciência, 1998. 602 p.

GRANADO-LORENCIO, C.; ARAUJO LIMA, C. R. M.; CERVIÁ, J. L. Abundance - distribution relationships in fish assembly of the Amazonas floodplain lakes. Ecography, Copenhagen, v 28, p. 515 $520,2005$.

GRANADO-LORENCIO, C.; LOBÓN CERVIA, J.; ARAUJO LIMA,C. R. M. Floodplain lake fish assemblages in the Amazon River: directions in conservation biology. Biodiversity and Conservation, New York, v. 16, p. 679-692, 2007.

HOEINGHAUS, D. J.; LAYMAN, C. A.; ARRINGTON, D. A.; WINEMILLER, K. O. Spatiotemporal variation in fish assemblage structure in tropical floodplain creeks. Enviromental Biology of Fishes, Dordrecht, v. 67, p. 379-387, 2003.

JUNK, W. J.; BAYLEY, P. B.; SPARKS, R. E. The flood pulse concept in river-floodplain ecosystems. In: DODGE, D. P. (Ed.). Proceedings of the International Large River Symposium. Ottawa: Canadian Special Publications in Fisheries and Aquatic Sciences, 1989. p. 110-127.

LATRUBESSE, E. M.; STEVAUX, J. C. Características físicobióticas e problemas ambientais associados à planície aluvial do Rio Araguaia, Brasil Central. Revista UnG Geociências, São Paulo, v. 5, n. 1, p. 65-73, 2006.

LEGENDRE, P.; LEGENDRE, L. Numerical ecology. Amsterdan: Elsevier, 1998. 853 p.

LIMA, J. D. Diversidade, estrutura trófica da ictiofauna e condições limnológicas em um lago na planície inundável do Rio das Mortes - Mato Grosso - Brasil. 2003. 110 f. Dissertação (Mestrado em Ecologia) - Universidade Federal de Mato Grosso, Cuiabá. 2003.

LIMA, J. D. 2009. Conectividade e análise da estrutura taxonômica e trófica da ictiofauna em lagos do Rio das Mortes - Mato Grosso - Brasil. 2009. 86 f. Tese (Doutorado em Ecologia) - Universidade Federal de São Carlos, São Carlos. 2009.

LIN, D. S. C.; CARAMASCHI E. P. Responses of the fish community to the flood pulse and siltation in a floodplain lake of the Trombetas River, Brazil. Hydrobiologia, Brussels, v. 545, p. 7591, 2005.

LOWE-MCCONNELL, R. H. Estudos ecológicos de comunidades de peixes tropicais. São Paulo: Editora da Universidade de São Paulo, 1999. 584 p.

MAGURRAN, A. E. Measuring biological diversity. Oxford: Blackwell Science, 2004. 256 p.

MELO, C. E.; LIMA, J. D. Diversidade de espécies e influência de fatores estocásticos na regulação da ictiofauna em lagos de meandro na Bacia do Rio das Mortes, Mato Grosso, Brasil. Revista SEB, São Carlos, v. 10, n. 2, p. 22-27, 2007.

MELO, C. E.; LIMA, J. D.; MELO, T. L.; PINTO-SILVA, V. Peixes do Rio das Mortes: identificação e ecologia das espécies mais comuns. Cuiabá: Central de Textos e Editora Unemat, 2005. 147 p.

MELO, C. E.; LIMA, J. D.; SILVA, E. F. Relationships between water transparency and abundance of Cynodontidae species in the Bananal floodplain, Mato Grosso, Brazil. Neotropical Ichthyology, Porto Alegre, v. 7, n. 2, p. 251-256, 2009. 
MELO, C. E.; MACHADO, F. A.; PINTO-SILVA, V. Diversidade de peixes em um córrego de cerrado no Brasil Central. Brazilian Journal of Ecology, São Paulo, v. 1, n. 2, p. 17-23, 2003.

MELO, T. L.; TEJERINA-GARRO, F. L.; MELO, C. E. Diversidade biológica da comunidade de peixes no baixo Rio das Mortes, Mato Grosso, Brasil. Revista Brasileira de Zoologia, Curitiba, v. 24, n. 3, p. 657-665, 2007.

MENESES, S. N. E. M.; COSTA, L. M.; COSTA, J. A. V. Os lagos do lavrado de Boa Vista - Roraima: Fisiografia, físico-química das águas, mineralogia, e química dos sedimentos. Revista Brasileira de Geociências, Curitiba, v. 37, n. 3, p. 478-489, 2007.

MÉRONA, B. Aspectos ecológicos da ictiofauna no baixo Tocantins. Acta Amazônica, Manaus, v. 16, n. 17, p. 109-124, 1986.

ODUM, E. P. Ecologia. Rio de Janeiro: Guanabara Koogan, 1998. $434 \mathrm{p}$.

PETRY, A. C.; AGOstinho, A. A.; GOMES, L. C. Fish assemblages of tropical floodplain lagoons: exploring the role of connectivity in a dry year. Neotropical Ichthyology, Porto Alegre, v. 1, n. 2, p. 111-119, 2003.

POUILLY, M.; RODRÍGUEZ, M. A. Determinism of fish assemblage structure in Neotropical floodplain lakes: influence of internal and landscape lake conditions. INTERNATIONAL SYMPOSIUM ON THE MANAGEMENT OF LARGE RIVERS FOR FISHERIES, II, 2004, Bangkok. Anais... Bangkok: FAO Regional Office for Asia and the Pacific, 2004. v. II. p. 243-265.

POUILLY, M.; YUNOKI, T.; ROSALES, C.; TORRES, L. Trophic structure of fish assemblages from Mamoré River floodplain lakes (Bolivia). Ecology of Freshwater Fish, Maiden, v. 13, n. 4, p. $245-$ 257, 2004.

RODRÍGUEZ, M. A.; LEWIS, W. M. Structure of fish assemblages along environmental gradients in floodplain lakes of the Orinoco River. Ecological Monographs, Washington, v. 67, n. 1,p. 109128, 1997.

SANTOS, G. M.; FERREIRA, E. J. G. Peixes da Bacia Amazônica. In: LOWE-MCCONNEL, R. H. (Ed.). Estudos ecológicos de peixes. São Paulo: EDUSP, 1999. p. 34-373.
SILVA, E. F.; MELO, C. E.; VÊNERE, P. C. Fatores que influenciam a comunidade de peixes em dois ambientes no baixo Rio das Mortes, planície do Bananal, Mato Grosso, Brasil. Revista Brasileira de Zoologia, Curitiba, v. 24, n. 2, p. 482-492, 2007.

SILVANO, R. A. M.; AMARAL, B. D.;OYAKAWA, O. T. Spatial and temporal patterns of diversity and distribution of the upper Juruá River fish community, Brazilian Amazon. Environmental Biology of Fishes, Dordrecht, v. 57, p. 25-35, 2000.

SIQUEIRA-SOUZA, F. K.; FREITAS, C. E. C. Fish diversity of floodplan lakes on the lower stretch of the Solimões River. Brazilian Journal Biology, São Paulo, v. 64, n. 3, p. 501-510, 2004.

SOUSA. R. C.; FREITAS. C. E. C. The influence of flood pulse ou fish communities of floodplain canals in the middle Solimões River. Neotropical Ichthyology, Porto Alegre, v. 6, n. 2, p. 249255, 2008.

SÚAREZ, Y. R.; PETRERE JÚNIOR, M.; CATELlA. C. C. Factors regulating diversity and abundance of fish communities in Pantanal lagoons Brazil. Fisheries Management and Ecology, East Yorkshire, v. 11 p. 45-50, 2004.

TEJERINA-GARRO, F. L.; R. FORTIN, R.; RODRÍGUEZ, M. A. Fish community structure in relation to environmental variation in floodplain lakes of the Araguaia River, Amazon Basin. Environmental Biology of Fishes, Dordrecht, v. 51, p. 399-410, 1998.

TONN, W. M.; MAGNUSON, J. J. Patterns in the species composition an richness of fish assemblages in northern Wisconsin lakes. Ecology, New York, v. 63, p. 1149-1166, 1982.

WINEMILLER, K. O.; TARIM, S.; SHORMANN, D.; COTNER, J. B. Fish assemblage structure in relation to environmental variation among Brazos River oxbow lakes. Transactions of the American Fisheries Society, New Jersey, v. 129, p. 451-468, 2000.

ZAR, J. H. Biostatistical analysis. New Jersey: Prentice Hall, 1996. $662 \mathrm{p}$. 